\title{
ERRATUM
}

\section{Entropy generation in parallel plate microchannels}

\section{Berrin Erbay · M. Murat Yalçın · Mehmet Ş. Ercan}

Published online: 8 February 2007

(C) Springer-Verlag 2007

Erratum to: Heat Mass Transfer

\section{DOI 10.1007/s00231-006-164-0}

Due to a processing error, the Eqs. 4 and 6 were missing. The correct equations are given below.

$$
\begin{aligned}
& R e=\frac{u_{0} D}{v}, \quad \operatorname{Pr}=\frac{v}{\alpha}, \quad x=\frac{x^{*}}{D}, \quad y=\frac{y^{*}}{D}, \quad \tau=\frac{t^{*} u_{0}}{D} \\
& u=\frac{u^{*}}{u_{0}}, \quad v=\frac{v^{*}}{u_{0}}, \quad \rho=\frac{\rho^{*}}{\rho_{0}}, \quad P=\frac{P^{*}}{\rho_{0} u_{0}^{2}} \\
& T=\frac{T^{*}-T_{\text {in }}}{T_{\text {wall }}-T_{\text {in }}} \\
& N_{s}=\dot{S}_{\text {gen }}^{\prime \prime \prime} \frac{D^{2}}{k \Omega^{2}}, \quad \phi=\frac{\mathrm{Br}}{\Omega}, \quad \mathrm{Br}=\frac{u_{0}^{2} \mu}{k \Delta T} \\
& \Omega=\frac{\Delta T}{T_{\text {in }}}, \quad \Delta T=T_{\text {wall }}-T_{\text {in }}
\end{aligned}
$$

The online version of the original article can be found at http://dx.doi.org/10.1007/s00231-006-0164-0.

L. B. Erbay $(\bowtie)$

Mechanical Engineering Department,

Eskisehir Osmangazi University, 26480 Eskisehir, Turkey

e-mail: lberbay@ogu.edu.tr

M. M. Yalçın

TUSAŞ Engine Industries, Inc., 26003 Eskisehir, Turkey

M. Ş. Ercan

Ford - Otosan İnönü Plant, 26140 Eskisehir, Turkey 